\title{
BMJ Open Multicomponent non-pharmacological intervention to prevent delirium for hospitalised people with advanced cancer: study protocol for a phase II cluster randomised controlled trial
}

To cite: Hosie A, Phillips J, Lam L, et al. Multicomponent non-pharmacological intervention to prevent delirium for hospitalised people with advanced cancer: study protocol for a phase II cluster randomised controlled trial. BMJ Open 2019:9:e026177. doi:10.1136/ bmjopen-2018-026177

- Prepublication history for this paper is available online. To view these files please visit the journal online (http://dx.do org/10.1136/bmjopen-2018026177).

Received 21 August 2018 Revised 26 November 2018 Accepted 28 November 2018

Check for updates

(c) Author(s) (or their employer(s)) 2019. Re-use permitted under CC BY-NC. No commercial re-use. See rights and permissions. Published by BMJ.

For numbered affiliations see end of article.

Correspondence to

Dr Annmarie Hosie;

annmarie.hosie@uts.edu.au

\begin{abstract}
Annmarie Hosie, ${ }^{1}$ Jane Phillips, ${ }^{1}$ Lawrence Lam, ${ }^{1}$ Slavica Kochovska, ${ }^{1}$ Beverly Noble, ${ }^{1}$ Meg Brassil, ${ }^{1}$ Susan E Kurrle, ${ }^{2}$ Anne Cumming, ${ }^{3}$ Gideon A Caplan, ${ }^{4,5}$ Richard Chye, ${ }^{6}$ Brian Le, ${ }^{7}$ E Wesley Ely, ${ }^{8}$ Peter G Lawlor, ${ }^{9}$ Shirley H Bush, ${ }^{9}$ Jan Maree Davis, ${ }^{10}$ Melanie Lovell, ${ }^{1,2,11}$ Linda Brown, ${ }^{1}$ Belinda Fazekas, ${ }^{1}$ Seong Leang Cheah, ${ }^{1}$ Layla Edwards, ${ }^{1}$ Meera Agar ${ }^{1}$
\end{abstract}

\section{Strengths and limitations of this study}

Introduction Delirium is a significant medical complication for hospitalised patients. Up to one-third of delirium episodes are preventable in older inpatients through non-pharmacological strategies that support essential human needs, such as physical and cognitive activity, sleep, hydration, vision and hearing. We hypothesised that a multicomponent intervention similarly may decrease delirium incidence, and/or its duration and severity, in inpatients with advanced cancer. Prior to a phase III trial, we aimed to determine if a multicomponent non-pharmacological delirium prevention intervention is feasible and acceptable for this specific inpatient group. Methods and analysis The study is a phase II cluster randomised wait-listed controlled trial involving inpatients with advanced cancer at four Australian palliative care inpatient units. Intervention sites will introduce delirium screening, diagnostic assessment and a multicomponent delirium prevention intervention with six domains of care: preserving natural sleep; maintaining optimal vision and hearing; optimising hydration; promoting communication, orientation and cognition; optimising mobility; and promoting family partnership. Interdisciplinary teams will tailor intervention delivery to each site and to patient need. Control sites will first introduce only delirium screening and diagnosis, later implementing the intervention, modified according to initial results. The primary outcome is adherence to the intervention during the first seven days of admission, measured for 40 consecutively admitted eligible patients. Secondary outcomes relate to fidelity and feasibility, acceptability and sustainability of the study intervention, processes and measures in this patient population, using quantitative and qualitative measures. Delirium incidence and severity will be measured to inform power calculations for a future phase III trial.

Ethics and dissemination Ethical approval was obtained for all four sites. Trial results, qualitative substudy findings and implementation of the intervention will be submitted for publication in peer-reviewed journals, and reported at conferences, to study sites and key peak bodies.
Trial registration number ACTRN12617001070325; Preresults.

Delirium is a serious acute neurocognitive disorder and medical complication for people with advanced cancer receiving palliative care in hospital, where it occurs for up to one in two patients and is reversible in only up to half of cases, at best. ${ }^{1-3}$ It causes sudden disruption to attention and cognition, such as memory and language deficit, disorientation and perception. ${ }^{1}$ During delirium, feelings of fear, humiliation, confusion and isolation are common, ${ }^{4}$ at a time when connection with family, friends and health professionals is important and highly valued..$^{5}$ Family experiences high levels of distress as a result. ${ }^{5}$ Delirium is further associated with increased falls, pressure areas, longer-term cognitive

\section{INTRODUCTION}


and functional decline, duration of hospital stay, mortality and healthcare costs. ${ }^{6-8}$

Despite the incidence of delirium and its profound impacts on people with advanced illness, there are limited treatment options and, to date, no effective pharmacological intervention. ${ }^{9-11}$ Nor have evidence-based processes for delirium prevention, recognition or assessment been translated in palliative care units. ${ }^{12} 13$ The most effective strategy for delirium in older patients across a range of hospital settings is prevention through non-pharmacological strategies to meet essential needs, such as physical and cognitive activity, sleep, hydration, vision and hearing. When implemented as a 'multicomponent intervention', these strategies have reduced delirium incidence by one-third. ${ }^{9}{ }^{14}$ A meta-analysis $(\mathrm{n}=4267)$ of randomised or matched trials of non-pharmacological prevention strategies reported significant reduction in delirium incidence, with the odds of delirium $53 \%$ lower in the intervention group compared with controls (OR $0.47,95 \%$ CI 0.38 to $0.58, \mathrm{p}<0.001) .{ }^{14}$ A Cochrane review of 39 randomised controlled trials (RCTs; $n=16082$ ) of non-pharmacological, medication or anaesthetic interventions reported that seven non-pharmacological intervention studies $(\mathrm{n}=1950)$ reduced delirium incidence (relative risk ( $\mathrm{RR}$ ) $0.69,95 \%$ CI 0.59 to 0.81 ), while evidence for most medication and anaesthetic interventions was uncertain. ${ }^{9}$ There was moderate quality evidence that the non-pharmacological interventions reduced length of hospital admission and improved the likelihood of return to independent living, with low-quality evidence of decreased delirium duration and severity. ${ }^{9}$ Studies of non-pharmacological interventions for delirium have mainly focused on older patients, yet often excluded patients with advanced cancer and other life-threatening illnesses. ${ }^{15}$ Also, strategies within the interventions were diverse, some were better operationalised than others and not all used a randomised design. ${ }^{14}$

The one study testing a non-pharmacological delirium prevention intervention for people with advanced cancer $(\mathrm{n}=1516)$ in seven Canadian specialist palliative care inpatient units reported no statistically significant difference in delirium incidence, total days in delirium, duration of first episode, severity or delirium-free survival. ${ }^{16}$ Strategies were fewer and less targeted to essential needs of patients than those reported in the more recent meta-analysis and Cochrane review ${ }^{914}$; and included (i) orientating patients to time, person and place each shift; (ii) informing family about delirium, its symptoms and prevention of confusion; and (iii) assessing pharmacological risk factors for delirium before querying physicians about consequent planned medication change. There also was inadequate rate and timeliness of completion of the primary measure, the Confusion Assessment Method. While adherence to the intervention was $>80 \%$, there was no difference in overall use of psychoactive medication between the two arms. Given that such medication is associated with delirium, ${ }^{17} 18$ this factor may partly explain the study's negative results. ${ }^{16}$
There are possible barriers to implementation of non-pharmacological delirium prevention strategies for people with advanced cancer. These include their common frailty and fatigue which reduces capacity to participate in activities such as exercise. Patients and family may not realise the serious risks associated with an episode of delirium or prioritise prevention strategies without this knowledge. Some clinicians may perceive that delirium is inevitable and innocuous in advanced cancer and palliative care contexts ${ }^{19}{ }^{20}$; and presume that preventing delirium is not possible, necessary or likely to be effective. Clinicians historically have relied on pharmacological intervention for delirium, rather than intentionally striving to prevent delirium through non-pharmacological means. With competing demands and without evidence of effectiveness, hospital managers may not value the importance of preventing delirium or allocate the required resources or personnel for non-pharmacological strategies, particularly for people approaching the end of their life.

Despite these barriers, the remit of palliative care to help patients live as actively as possible makes it important to study whether delirium can be prevented during advanced cancer. Based on the body of research conducted with older people in hospital, ${ }^{914}$ we hypothesised that a similar multicomponent intervention would reduce delirium incidence and/or decrease its duration and severity for this inpatient population. Given the above-noted possible challenges to implementation, piloting the intervention and study design was required prior to testing the hypothesis in a phase III (efficacy) trial.

\section{AIM AND OBJECTIVES}

To determine if a multicomponent non-pharmacological delirium prevention intervention is feasible and acceptable for inpatients with advanced cancer.

The objectives are to:

1. Develop a multicomponent non-pharmacological delirium prevention intervention ("non-pharmacological delirium prevention intervention'), derived from highly efficacious interventions for older adults in hospital, for people with advanced cancer and palliative care inpatient unit settings.

2. Describe the strategies used by participating sites to implement the delirium measurement tools and non-pharmacological delirium prevention intervention.

3. Determine if a non-pharmacological delirium prevention intervention is feasible, acceptable and deliverable with high adherence and fidelity in oncology and palliative care units.

4. Determine the feasibility and design of a phase III trial to test the efficacy of the non-pharmacological delirium prevention intervention in people with advanced cancer in hospital. 


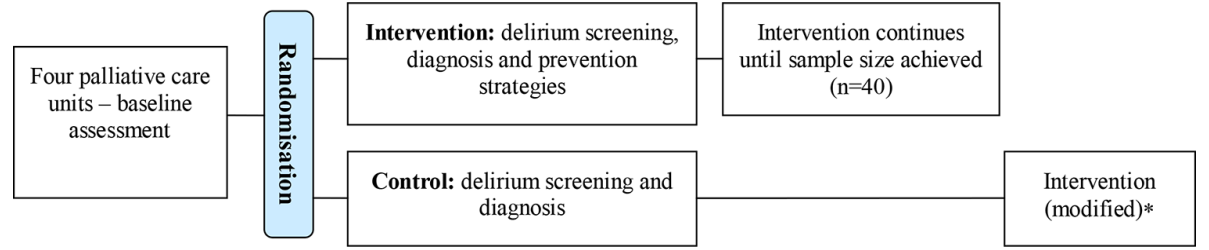

Figure 1 Study diagram of standardised delirium screening at all sites+delirium prevention strategies at intervention sites; control sites waitlisted for intervention. *Modified if required.

\section{METHODS AND ANALYSIS \\ Design}

A phase II, cluster RCT with a waitlist control. ${ }^{21}$ Participating sites will be randomised to the intervention (screening and immediate implementation of intervention) or control (screening and waitlist to the modified intervention) (figure 1).

The use of this design in the phase II trial was to inform the feasibility and design, delivery methods and power calculations of a future multisite phase III cluster RCT. A cluster approach was chosen because the proposed intervention is more suited to implementation at a site level, and a traditional RCT design would risk contamination in the control arm. The use of a cluster RCT design is an advance on prior studies of non-pharmacological prevention interventions that used non-randomised designs. A waitlist control arm was chosen as key stakeholders at interested sites considered that the delirium prevention strategies were important; that participation in a trial that enabled access to the intervention was more appealing and ethically sound; and that the intervention strategies were well established as effective in other hospital settings and the potential benefits were clear, in principle. The waitlist control adds to the resource and time requirements of the trial, but will allow the intervention and study processes to be modified and/or refined at the two waitlist control sites, should initial results indicate that this is required. ${ }^{21}$

\section{Sites (clusters) and patient population}

The participating sites are four Australian palliative care units, where approximately $75 \%$ of patients have a primary diagnosis of advanced cancer. ${ }^{22}$

In line with the cluster RCT design, consent to participate was obtained at the site level from the person with the delegation to approve participation. Data will be collected for all admitted patients aged $\geq 18$ years with a diagnosis of advanced cancer, for which no individual patient consent will be required.

\section{Intervention}

Intervention sites will implement (i) delirium screening, (ii) delirium diagnosis assessments and (iii) the multicomponent delirium prevention intervention.

Bedside nurses will undertake the Nursing Delirium Screening Scale (Nu-DESC ${ }^{23}$ for all eligible patients at the end of every shift. Within 24 hours of the patient assessed as having a Nu-DESC score $\geq 2$, a trained physician will apply Diagnostic and Statistical Manual of
Mental Disorders, Fifth edition (DSM-5) diagnostic criteria for delirium, ${ }^{1}$ operationalised using the Delirium Rating Scale-Revised-1998 (DRS-R-98) ${ }^{24}$ These processes currently are not routine at the participating sites and therefore will be additional to usual care.

The multicomponent delirium prevention intervention involves five domains of care that, when delivered in combination, significantly reduced delirium incidence in older hospitalised patients in previous clinical trials. ${ }^{9} 14$ We added family partnership as the sixth domain as it was recommended by our consumer investigators and an expert working group, is highly valued by patients and family members, ${ }^{525}$ and has been identified as essential by the Australian Commission for Safety and Quality in Healthcare in a new Delirium Standard, if preferred by the patient. ${ }^{26}$ We did not include a pharmacological component (such as minimising polypharmacy) because there was less evidence that this effectively prevents delirium compared with clinical care that which addresses fundamental human needs for physical and cognitive activity, sleep, hydration, vision and hearing. ${ }^{9,14}$

The delirium prevention intervention will be delivered to all eligible patients for the first seven days of admission by members of the interdisciplinary team, family caregivers and volunteers. The domains and strategies of the multicomponent intervention are presented in table 1 .

Control sites will initially implement only delirium screening and diagnosis. Once the intervention sites achieve their sample, control sites will implement the intervention.

All sites will continue usual care with respect to treatment of patients with delirium.

\section{Site engagement, education and training}

The phase II trial will not predetermine delivery methods for the intervention, instead observing the methods of each site in order to learn about their team's established practice, as well as what practices they needed to establish. Engagement of site staff and volunteers will be guided by Michie's Behaviour Change Wheel, an evidenced-based framework for changing health-related behaviours. ${ }^{27}$ Each site will form an interdisciplinary working group of medical, nursing, allied health, pastoral care, volunteer coordinator and managerial staff. The function of the working groups will be to determine how to deliver the intervention with the available resources, composition and capabilities of their site team. ${ }^{27}$ Working group members will communicate the study to the whole team, 
Table 1 Multicomponent delirium prevention intervention

\begin{tabular}{|c|c|c|}
\hline Domain & Strategies & Implementation \\
\hline $\begin{array}{l}\text { Preserve natural } \\
\text { sleep }\end{array}$ & $\begin{array}{l}\text { Offer ear plugs to patients with low risk of falls } \\
\text { Offer eye shades to patients with low risk of falls } \\
\text { Reduce noise outside patient rooms during } 21: 00-06: 00 \\
\text { Normal day-night light variation in room and unit } \\
\text { Exposure to natural light during daylight hours } \\
\text { Schedule care activities to allow uninterrupted sleep during } \\
\text { the night } \\
\text { Avoid caffeine after } 16: 00\end{array}$ & $\begin{array}{l}\text { The patient wears ear plugs at night } \\
\text { The patient wear eye shades at night } \\
\text { Room curtains/blinds are open during the day } \\
\text { Room lights are off or minimised at night } \\
\text { The patient spends time outside during the day } \\
\text { The patient drinks no caffeinated drinks after } 16: 00 \\
\text { The patient reports uninterrupted night-time sleep }\end{array}$ \\
\hline $\begin{array}{l}\text { Promote } \\
\text { communication, } \\
\text { orientation and } \\
\text { cognition }\end{array}$ & $\begin{array}{l}\text { Interpreter and translation for people of non-English speaking } \\
\text { background } \\
\text { Greet the patient by name } \\
\text { Introduce self by name and role } \\
\text { Refer to person, time and place when talking with the patient } \\
\text { Time aids in room for example, watch, personal or wall clock; } \\
\text { wall, desk or electronic calendar } \\
\text { Update in-room whiteboards daily with date, day, place, } \\
\text { reason for admission, team member names, schedule } \\
\text { Minimise number of transfers to other beds or rooms within } \\
\text { the unit } \\
\text { Discuss current events with the patient } \\
\text { Encourage the patient to reminisce and talk } \\
\text { Encourage the patient to engage in cognitively stimulating } \\
\text { activities }\end{array}$ & $\begin{array}{l}\text { Interpreter is available and used } \\
\text { Orientating information is translated into the } \\
\text { patient's native language } \\
\text { The patient can see the time, day, date and month } \\
\text { in their room } \\
\text { The patient remains in the same bed location within } \\
\text { the unit } \\
\text { The patient discusses current events } \\
\text { The patient reminisces and/or talks about their life } \\
\text { and family } \\
\text { The patient spends time in cognitively stimulating } \\
\text { activities, for example, reading, puzzles, games, } \\
\text { knitting, music } \\
\text { Cognitive stimulating activities are in the patient's } \\
\text { care plan }\end{array}$ \\
\hline Family partnership & $\begin{array}{l}\text { Ask family about the patient's baseline cognition } \\
\text { Inform the patient and family about delirium risk } \\
\text { Inform the patient and family about delirium prevention } \\
\text { strategies and invite participation }\end{array}$ & $\begin{array}{l}\text { Family are asked about the patient's baseline } \\
\text { cognition on admission } \\
\text { Delirium information brochure is provided to the } \\
\text { patient and family } \\
\text { Verbally inform of delirium risk and prevention } \\
\text { Patients and family are invited to participate in } \\
\text { delirium prevention strategies }\end{array}$ \\
\hline
\end{tabular}

promote the delirium screening, diagnosis and prevention strategies, and inform patients and family about delirium and the prevention strategies. Site teams will be encouraged to tailor the intervention strategies to each patient's assessed needs and preferences to ensure person-centred care, as well as to adopt simple and feasible methods of delivery and documentation of the intervention.

Education and training of site staff and volunteers in the delirium screening and prevention strategies will be standardised, interdisciplinary and based on Biggs' 
educational model. ${ }^{28}{ }^{29}$ This model will align educational objectives and methods with the delirium learning needs of staff, and promote critical reflection on attitudes, practice and functional knowledge of the complexities of caring for a person with advanced cancer in hospital. ${ }^{28} 29$ Education and training will take place for 2 months prior to data collection. A brief, simple study overview manual also will be developed.

Study investigators and/or project staff will attend sites to (i) promote fidelity to the study processes and aims; (ii) assist with education and training activities; (iii) resolve issues that delay implementation of the intervention or threaten its integrity; (iv) act as a 'delirium resource person' and (v) support and encourage site staff and volunteer participation in the intervention.

The frequency, duration and mode of administration of education and training will be determined prior to implementing delirium screening, diagnosis and prevention strategies in collaboration with participating sites, then standardised for each. Based on the learnings obtained in this phase II trial, we will develop a replicable standardised education resource for the phase III trial.

\section{Randomisation}

In keeping with the method of the anticipated phase III trial, we will use a permuted block randomisation method with various block sizes to allocate sites to the intervention or waitlist control. Randomisation will be performed by the study statistician (LL) from the coordinating centre, the University of Technology Sydney (UTS).

\section{Blinding and avoidance of contamination}

The study design and nature of the intervention means that blinding of site staff will not be possible. Written information for patients and family caregivers will provide only general information about the study aims, rather than specifics of the design or site allocation. Attention will be focused on research nurse training and standardisation of data collection to limit the potential for bias.

To avoid contamination between sites, personnel collecting data at an intervention site will not collect data in a control site, and vice versa. Site investigators, research nurses and project staff will be asked not to discuss the intervention in joint tele-meetings with control sites. Clinicians at control sites initially will receive information and training on delirium screening and diagnosis only, and only general information about the prevention intervention in discussions and promotion, until they move into the intervention phase.

\section{Data collection}

Research nurses will collect baseline data from sites' most recent Palliative Care Outcomes Collaborative (PCOC) report (a national programme which measures and benchmarks patient outcomes in palliative care using standardised clinical assessment tools) ${ }^{23}$ (figure 2) and from key personnel. Research nurses will screen consecutively admitted patients for eligibility, collect delirium screening and diagnostic assessment measures for enrolled patients and record these in a Case Report Form (CRF). At intervention sites, specially designed checklists will capture family caregivers, staff and volunteers' delivery of delirium prevention strategies within each domain of the multicomponent intervention (table 1), as well as who delivered it. From this, we will determine the level of involvement of family caregivers, interdisciplinary staff and volunteers for each strategy and domain. Whenever the patient does not receive the strategy, the reason will be recorded according to the following categories:

- Not required

- Patient choice

- Not clinically appropriate

- Not possible with current resources

- Other

At study completion, the project team will collect PCOC data for the study time frame (age, gender, country of birth, preferred language, Aboriginal or Torres Strait Islander status, primary diagnosis, length of stay, performance status (Australian-modified Karnofsky Performance Status (AKPS) ${ }^{30}$ and Resource Utilisation Groups-Activities of Daily Living (RUG-ADL)), ${ }^{31}$ palliative care phase).$^{32}$ For the sustainability outcome, site research nurses will collect intervention adherence data at 6 months for all inpatients for 1 week.

\section{Assessments}

Figure 2 gives the schedule of study measures and time points; Box 1 provides information on the palliative care and delirium measures.

\section{Outcomes}

The primary outcome is adherence to the intervention. A rate of at least $60 \%$ of patients having at least four completed domains for at least five of the first seven days of admission will be considered minimum evidence that the intervention is feasible without need for major modification of the intervention or its delivery methods. Endpoints will be at completion of the intervention and modified intervention arms (figure 1).

We chose this moderate endpoint because of the potential patient, clinician and system-level challenges to the non-pharmacological strategies in the context of advanced cancer. Consensus by investigators was this endpoint would be the minimum to still have impact, realistic to achieve in practice, and ensure that further evaluation of this complex intervention was not prematurely stopped. The waitlist control design will allow two endpoints and thereby maximise the potential to reach this level of adherence to the intervention.

Secondary outcomes will further inform of the feasibility, acceptability and potential efficacy of a phase III trial of the intervention in this patient population and setting, as follows:

1. Coverage: Delivery rate of the multicomponent intervention to consecutive eligible patients admitted to 


\begin{tabular}{|c|c|c|c|c|c|c|c|}
\hline \multirow[t]{3}{*}{ Measures } & \multicolumn{7}{|c|}{$\begin{array}{l}\text { Study period } \\
\end{array}$} \\
\hline & \multicolumn{5}{|c|}{ Control and intervention sites } & \multicolumn{2}{|c|}{ Intervention sites } \\
\hline & Baseline & $\begin{array}{l}\text { Eligibility } \\
\text { screen on } \\
\text { admission } \\
\end{array}$ & $\begin{array}{l}\text { Admission } \\
\text { days 1-7 }\end{array}$ & $\begin{array}{l}\text { Nu- } \\
\text { DESC } \\
+ \text { ve }\end{array}$ & $\begin{array}{l}\text { Study } \\
\text { completion }\end{array}$ & $\begin{array}{l}\text { Admission } \\
\text { days 1-7 }\end{array}$ & $\begin{array}{l}\text { Intervention } \\
\text { completion }\end{array}$ \\
\hline \multicolumn{8}{|l|}{ UNIT LEVEL } \\
\hline $\begin{array}{l}\text { Geographical } \\
\text { location }\end{array}$ & $\mathrm{X}$ & & & & & & \\
\hline $\begin{array}{l}\text { Type and level of } \\
\text { service provision }\end{array}$ & $\mathrm{X}$ & & & & & & \\
\hline Number of beds & $\mathrm{X}$ & & & & & & \\
\hline Team composition & $\mathrm{X}$ & & & & & & \\
\hline $\begin{array}{l}\text { Clinical } \\
\text { documentation } \\
\text { method }\end{array}$ & $\mathrm{X}$ & & & & & & \\
\hline $\begin{array}{l}\text { Delirium process } \\
\text { and measures }\end{array}$ & $\mathrm{X}$ & & & & & & \\
\hline $\begin{array}{l}\text { Patient } \\
\text { demographics* }\end{array}$ & $\mathrm{X}$ & & & & $\mathrm{X}$ & & \\
\hline $\begin{array}{l}\text { Patient function } \\
\text { AKPS, RUG- } \\
\text { ADL* }\end{array}$ & $\mathrm{X}$ & & & & $\mathrm{X}$ & & \\
\hline $\begin{array}{l}\text { Palliative care } \\
\text { phases* }\end{array}$ & $\mathrm{X}$ & & & & $\mathrm{X}$ & & \\
\hline \multicolumn{8}{|l|}{$\begin{array}{l}\text { PATIENT } \\
\text { LEVEL }\end{array}$} \\
\hline Primary diagnosis & & $\mathrm{X}$ & & & & & \\
\hline Age & & $\mathrm{X}$ & & & & & \\
\hline $\mathrm{Nu}-\mathrm{DESC}$ & & & $\mathrm{X}$ & & & & \\
\hline $\begin{array}{l}\text { DSM-5 diagnostic } \\
\text { criteria for } \\
\text { delirium }\end{array}$ & & & & $\mathrm{X}$ & & & \\
\hline DRS-R-98 & & & & $\mathrm{X}$ & & & \\
\hline $\begin{array}{l}\text { Adherence to } \\
\text { delirium } \\
\text { prevention } \\
\text { strategies }\end{array}$ & & & & & & $\mathrm{X}$ & $\begin{array}{l}\mathrm{X} \text { (at six } \\
\text { months) }\end{array}$ \\
\hline \multicolumn{8}{|l|}{ SUB-STUDY } \\
\hline $\begin{array}{l}\text { Brief interviews } \\
\text { with patients, } \\
\text { family, staff and } \\
\text { volunteers }\end{array}$ & & & & & & & $\mathrm{X}$ \\
\hline
\end{tabular}

Figure 2 Schedule of study measures and time points. ${ }^{43}$ Characteristics indicated with a * will be collected at baseline from the sites most recent Palliative Care Outcomes Collaborative (PCOC) report, and then again at study completion directly from PCOC for the specific time frame of data collection at each site. AKPS, Australian-modified Karnofsky Performance Status; DRS-R-98, Delirium Rating Scale-Revised-1998; DSM-5, Diagnostic and Statistical Manual of Mental Disorders, Fifth edition; Nu-DESC, Nursing Delirium Screening Scale; RUG-ADL, Resource Utilisation Groups-Activities of Daily Living.

the unit, reasons why the intervention was not delivered, weekend coverage, measured via screening logs and CRFs.

2. Fidelity to delirium screening, diagnosis and the intervention: Degree of alignment with the protocol, rationales for adaptation, rate of protocol deviations without reasons, measured via CRFs.

3. Methods, areas and levels of interdisciplinary involvement in delivery of the intervention, measured via intervention checklist.

4. Feasibility and acceptability of the study intervention and measures for patients, caregivers, staff and volunteers, measured via brief interviews during and shortly after the intervention phase.
5. Sustainability of the intervention: Adherence will be measured for all inpatients over 1 week, 6 months after commencement of data collection at the intervention sites.

6. Feasibility of the sample: Percentage of participants included in data collection, reasons for non-inclusion, time to achieve sample size, measured via screening logs and CRFs.

7. Number of people with advanced breast cancer admitted to the units, number of these who are in underserved populations (patients over 70, indigenous patients and culturally and linguistically diverse backgrounds), and the number who experience an episode of delirium (total, and in underserved 


\section{Box 1 Description of study measures}

The Australian-modified Karnofsky Performance Status was adapted from the Karnofsky Performance Status with good face validity and longitudinal test-retest reliability. ${ }^{30}$ The AKPS measures patients' overall performance status, using 10-point increments along a scale of $100-10$. A score of 100 denotes normal function with no evidence of disease, decreasing to a minimum score of 10 , assigned when patients are comatose or barely rousable. Routinely applied on an at least daily basis in most Australian inpatient unit palliative care services. The AKPS will be used to report the patient cohort's performance status at participating sites.

The Resource Utilisation Groups-Activities of Daily Living ${ }^{31}$ is a validated functional assessment tool which assigns a score of 4-18, based on what a patient does in relation to bed mobility, transfers, eating and toileting, rather than they can do. Higher scores indicate the need for more assistance to undertake activities and that more resources are required to provide this assistance. Applied on an at least daily basis in most Australian inpatient unit palliative care services. The measure will be used to report the patient cohort's functional status at participating sites.

The Palliative Care Phase ${ }^{32}$ classification is not a validated tool, but is applied on an at least daily basis in most Australian palliative care services to describe the needs of the patient and family and prompt a timely and appropriate clinical response. Phases are (i) stable (problems and symptoms are adequately managed and there is a plan of care); (ii) unstable (urgent intervention required because a new symptom or problem develops or an existing problem rapidly escalates); (iii) deteriorating (a gradual decline in function and worsening of an existing problem or development of a new but anticipated problem); (iv) terminal (death is likely within days); and (v) bereavement (post death support). The measure will be used to report the patient cohort's palliative care needs at participating sites.

The Nursing Delirium Screening Scale ${ }^{24}$ was validated in an oncology inpatient population with a sensitivity of $85.7 \%$ and specificity of $86.8 \%$. ${ }^{24}$ It is a brief (<1 min) five-item and low burden tool, incorporating nurses' observation of disorientation, inappropriate behaviour, inappropriate communication, illusions/hallucinations and psychomotor retardation. Nurses assign a score of 0-2 for each item, giving a maximum score of 10 . The psychomotor retardation item improves recognition of hypoactive delirium, ${ }^{33}$ the most prevalent subtype in palliative care inpatient populations. ${ }^{3}$ The Nu-DESC has been used in previous research in inpatient palliative care populations ${ }^{11}$ and was considered feasible and acceptable by palliative care nurses. ${ }^{19}$ The Nu-DESC will be used by bedside nurses to screen patients for delirium every 8-hour shift.

The Diagnostic and Statistical Manual of Mental Disorders, Fifth edition diagnostic criteria for delirium are within the most current version of the American Psychiatric Association: Diagnostic and Statistical Manual of Mental Disorders. ${ }^{1}$ Criteria are (i) Disturbed attention and awareness; (ii) disturbance developed over a short period of time (usually hours to a few days), is a change from baseline attention and awareness and fluctuates in severity; (iii) an additional disturbance in cognition; (iv) disturbances in i and iii are not caused by another neurocognitive disorder nor occur in the context of severely reduced level of arousal; and (v) the disturbance is a direct physiological consequence of another medical condition, substance intoxication or withdrawal, exposure to a toxin or has multiple aetiologies. Treating physicians will use the DSM- 5 to determine a delirium diagnosis.

The Delirium Rating Scale-Revised- $98^{25}$ is a 16 -item delirium severity and diagnostic scale with scores of up to 46 . It had high inter-rater reliability, sensitivity and specificity in the original validation study, ${ }^{25}$ high sensitivity and adequate internal consistency and factor validity in patients with cancer, ${ }^{34}$ and has been used in research with palliative care inpatients. ${ }^{356}$ The DRS-R-98 was designed to measure a wider range of delirium symptoms than are contained within diagnostic criteria and in different settings had good discriminative capacity for all, including in a patient population with a high prevalence of dementia. ${ }^{37} 38$ Severity items are sleep-wake cycle disturbance; perceptual disturbances and hallucinations; delusions; lability of affect; language; thought process abnormalities; motor agitation; motor retardation; orientation; attention; short-term memory; long-term memory; and visuospatial ability. Diagnostic items are temporal onset of symptoms; fluctuation of symptom severity; physical disorder. Information is obtained from all sources, including physical examination, history gathering and formal cognitive testing. Requires clinician training, with guidance for use contained within the tool. Trained treating physicians and nurses will use the DRS-R-98 to operationalise delirium diagnosis and measure delirium severity. We will use a diagnostic cut-off score of $>15^{38}$

populations) (for the purposes of reporting to the trial funder, the National Breast Cancer Foundation).

8. Percentage completion of all study measures, measured via CRF.

9. Rate of patients with a positive delirium screen, measured according to a score of $\geq 2$ on the Nu-DESC at least once during each 24 hours period.

10. Delirium incidence, measured at first onset according to the DSM-5 diagnostic criteria for delirium applied within 24 hours of a positive delirium screen.

11. Delirium severity measured at first onset, using the DRS-R-98.

12. Number of falls, complaints and other adverse events related to the intervention.

\section{Substudy}

A qualitative substudy will be conducted to obtain patient, family caregiver, staff and volunteer perceptions of the feasibility and acceptability of the intervention strategies (eg, receiving information from staff about delirium) and study measures via brief, semistructured interviews (figure 2).

\section{Inclusion and exclusion criteria for the substudy}

1. Patients will be included if they are aged 18 years or older; have a diagnosis of advanced cancer; admitted to an intervention site and received the intervention; speak English or have access to a healthcare interpreter; and able to give fully informed written consent. Patients with advanced breast cancer will be purposively recruited to participate in the interviews. Patients will be excluded if they have an $\mathrm{AKPS}^{30}$ score $<30$ and are in the 'terminal' Palliative Care Phase. ${ }^{32}$

2. Family caregivers will be included if they are aged 18 years or older; identified as a caregiver of a patient who received the intervention; English speaking or have availability of a healthcare interpreter and able to give fully informed written consent. 
3. Site staff will be included if they are employed at an intervention site and involved in implementing the delirium measures and/or the intervention.

4. Site volunteers will be included if they are aged 18 years or older, enrolled in a formal volunteer programme at an intervention site and involved in implementing the intervention.

\section{Substudy consent process}

A researcher who is not a study investigator will obtain written informed consent from patients, family caregivers, staff and volunteers to participate in the brief interviews. For patients and family caregivers, the researcher will check with the clinical team to make sure the person meets the broad criteria for consideration of eligibility, is well enough and has given permission to be approached by a researcher, before introducing himself or herself to the person and explaining the study. For staff and volunteers, the researcher will consult with the site investigator before approaching potential participants.

Participant consent will be a process of information exchange between the researcher, the potential participant and any other person the potential participant believes should be included in the discussion. Participant information sheets will be the basis for discussion and cover all procedures and possible benefits and burdens of participating. The potential participant will be given sufficient opportunity to consider the study and ask questions. Any questions will be addressed and answered fully. The completed consent form will be copied and one copy will be given to the participant, one copy inserted in the medical file (for patients) and one copy filed in study file.

\section{Analysis}

\section{Statistical analysis of primary outcome (adherence)}

Adherence will be calculated as the rate to which patients have completed domains on a daily basis for the first sevendays of admission. Degree of adherence to individual strategies will also be calculated as proportions.

\section{Statistical analysis of secondary outcomes}

Data on all outcomes will be summarised with descriptive statistics including their distribution. Frequency and percentage will be used for summarising categorical variables and mean, SD, median and IQR for continuous variables. Delirium incidence and severity will be determined at both the intervention and control sites.

\section{Qualitative analysis}

Participant interviews will be analysed using thematic content analysis to identify emergent themes and trends related to participants' perceptions of the feasibility and acceptability of the intervention elements and delirium measures. ${ }^{39}$

\section{Sample size}

A sample size of four sites and 40 patient participants (10 from each site) was considered sufficient for reasonable estimation of feasibility and percentage completion of study processes and measures during the first phase. ${ }^{40}$ We will collect deidentified data on all eligible patients admitted to all sites until data are collected for 40 patients overall, with at least 20 in the intervention arm. If the intervention is found to need modification, data will be collected for a further 20 patient participants at the two waitlist control sites.

This sample size was based on that projected for the future phase III cluster RCT of the intervention with: two parallel arms, $50 \%$ delirium incidence in the control, $30 \%$ delirium incidence in the intervention group, cluster size of 30 and intraclass correlation of 0.05 , type I error rate of $5 \%, 80 \%$ power to reject the null hypothesis and $30 \%$ attrition. This calculation results in a projected phase III trial sample size of nine clusters and 280 patient participants.

For the substudy, sample size will be determined when data saturation is achieved.

\section{Trial monitoring}

In addition to falls and complaints, all adverse events will be recorded. Site investigators will assess the adverse event, assign the degree of relationship to the intervention and provide information to the coordinating centre (UTS), and the approving Human Research Ethics Committee (HREC) if required. Adverse events will be followed until the event is resolved, can be explained or if the participant is lost to follow-up. Reports will contain details of follow-up investigations, results or other consultation. The investigator team will stop the study if reporting of adverse events indicates that major review of the study protocol is required. The UTS project team will report adverse event related to the intervention to the PaCCSC Trial Management Committee (TMC) within 2 weeks of knowledge of the event. The TMC discussions will be minuted, with actions detailed and reviewed at the subsequent meeting. The TMC chairperson's report to the PaCCSC Scientific Committee will contain a summary of the discussions of the adverse event report and agreed outcomes.

\section{Data management}

An Excel spreadsheet master index will contain confidential participant contact information and be the only link between individual site and patient participants and their allocated identification number (ID). Study data will be collected and stored on paper CRFs and electronic Excel spreadsheets and then entered onto and managed on a Research Electronic Data Capture (REDCap) ${ }^{41}$ database. Audio data from participant interviews will be identified only by ID, collected on a digital recording medium and stored temporarily at the study sites until uploaded to the REDCap database. Original files will then be destroyed. Data will be held, administered, checked and analysed at the coordinating site according to relevant PaCCSC Standard Operating Procedures. Errors detected during the data checking process will generate a site data report form recording details of the query and correction and resolution instructions. The database will be updated 
according to site instructions via email to provide an audit trail of data changes. The coordinating site will maintain a register of data checks for monitoring purposes. Data collected at each site, such as CRFs, any corrected and amended data, copies of adverse incident reports and file notes, will be securely stored and identified by ID number only. All identifiable data (eg, signed consent forms) will be separately stored during the recruitment period. Site research staff will send copies of study documents (with the exception of signed consent forms) to the coordinating site by registered mail for collation and archiving. All study documents will be stored in accordance with relevant state government regulations regarding the retention and disposal of participant records.

\section{Patient and public involvement}

The study rationale and processes were informed by the literature pertaining to patients' experiences of delirium, as outlined in the introduction. ${ }^{45}$ Low-burden outcome measures, such as the Nu-DESC, were deliberately chosen in order to minimise the impact of the study on patients with advanced illness. No patients were directly involved in the design, recruitment to or conduct of the study. Two family caregiver consumers are associate investigators of the study ( $\mathrm{MB}$ and $\mathrm{BN})$. We will include the perspectives of patients about the feasibility and acceptability of the intervention through brief semistructured interviews. Investigators will not have access to the names or contact information of patient or family caregiver participants in order to directly provide feedback about the study to them. At study completion, a written and verbal report of the results and findings will be provided to the participating sites.

\section{Ethics and dissemination}

The study was approved by the South Western Sydney Local Health District HREC on 19 July 2017, reference number HREC/17/LPOOL/224; and ratified by the UTS HREC on 22 August 2017, reference number ETH171697. Minor protocol amendments were approved on 13 April 2018 (V1.1).

Reporting of this protocol adheres to the Standard Protocol Items: Recommended for Interventional Trials. ${ }^{42}$ Reporting of results will adhere to the Consolidated Standards of Reporting Trials guidelines for cluster RCTs and non-pharmacological treatment trials. ${ }^{43}$ Reporting of the qualitative substudy and implementation findings will be guided by the Consolidated Criteria for Reporting Qualitative Research. ${ }^{45}$ A comprehensive dissemination strategy will ensure that the trial results (either positive or negative) inform future research and clinical practice. Dissemination will include publication in peer-reviewed journals, presentations at conferences, study sites and key peak bodies. The investigators have no publication restrictions.

\section{Strengths and limitations}

The primary strengths of this study are the cluster RCT design and that it is supported by the PaCCSC, a national, multisite phase III clinical trials group which provides well-established rigorous research governance and access to sites with research experience and capacity. The intervention includes family partnership, which is highly valued by both patients and family. ${ }^{526}$ We will obtain the perspectives of patients and family, which are largely absent in previous trials of multicomponent delirium interventions. ${ }^{15}$

Limitations include that site and research staff will not be blinded to the intervention. Active steps will be taken to minimise contamination between intervention and waitlist control sites. The study will be conducted in Australian palliative care inpatient settings and include only patients with advanced cancer, limiting the generalisability of results for services in other geographical regions and healthcare systems, and for patients with other advanced illnesses.

\section{Trial status}

The study has been approved by local health district and university HRECs, local governance approvals obtained, sites randomised, the 2-month training period completed and data collection is under way.

\section{Author affiliations}

${ }^{1}$ IMPACCT - Improving Palliative, Aged and Chronic Care through Clinical Research and Translation, University of Technology Sydney, Sydney, New South Wales,

Australia

${ }^{2}$ Hornsby Ku-ring-gai Health Service, Northern Clinical School, University of Sydney, Hornsby, New South Wales, Australia

${ }^{3}$ Australian Commission on Safety and Quality in Healthcare, Sydney, New South Wales, Australia

${ }^{4}$ Geriatric Medicine, Prince of Wales Hospital, Sydney, New South Wales, Australia ${ }^{5}$ University of New South Wales, Randwick, New South Wales, Australia

${ }^{6}$ Sacred Heart Health Service, St. Vincent's Hospital, Darlinghurst, New South Wales, Australia

${ }^{7}$ Palliative and Supportive Services, Royal Melbourne Hospital, Melbourne, Victoria, Australia

${ }^{8}$ Critical IIIness, Brain Dysfunction, and Survivorship (CIBS) Center at Vanderbilt University, and the Tennessee Valley Veteran's Affairs Geriatric Research Education Clinical Center (GRECC), Nashville TN USA, Nashville, Tennessee, USA

${ }^{9}$ Department of Medicine, University of Ottawa, Ottawa, Ontario, Canada

${ }^{10}$ Palliative Care, Calvary Health Care Kogarah, Sydney, New South Wales, Australia

${ }^{11}$ HammondCare, Greenwich Hospital, Greenwich, New South Wales, Australia

Acknowledgements The authors gratefully acknowledge Associate Professor Andrew Teodorczuk, Dr Aileen Collier, Ms Bronwyn Heron and Dr Christine Sanderson who contributed clinical and research expertise to the development of the non-pharmacological delirium prevention intervention.

Contributors Chief study investigators MA, AH and JP retain ultimate responsibility for the trial. Investigators and a project team coordinated the trial from Improving Palliative, Aged and Chronic Care through Clinical Research and Translation, UTS. The investigator team meet at least twice yearly to support progress of the trial and inform related activities, such as dissemination. AH, MA and JP are the co-lead authors. MA, JP and AH devised the adaptation of the multicomponent intervention for people with advanced cancer in hospital. MB and BN provided consumer insight into the adaptation of the intervention. AC provided guidance on the extent of alignment of the intervention and delirium screening diagnosis processes with the ACSQHC Delirium Clinical Care Standard. LL devised the statistical analysis and randomization process. JMD and ML provided insights into the waitlist design. SK contributed to the development of the site engagement and educational processes. SEK, GC, RC, BL, EWE, PL and SHB contributed clinical and research expertise into study design, process, measures and/or analysis. LB, BF, SLC and LE contributed to various aspects of the study protocol, including data collection, entry and storage, reporting of adverse effects, minimisation of contamination and/or site training. All authors have read and approved the final manuscript. 
Funding This work was supported by an Australian National Breast Cancer Foundation (NBCF) 2017 Pilot Study Grant (grant code PS-17-030). The trial sponsor is PaCCSC. PaCCSC supports optimal trial governance through SOPs for electronic data handling, completion of CRFs, monitoring, dissemination, archiving of research materials and record destruction; and trial infrastructure through Trials Management and Scientific Committees.

Competing interests None declared.

Patient consent for publication Not required.

Ethics approval South Western Sydney Local Health District (SWSLHD) HREC.

Provenance and peer review Not commissioned; externally peer reviewed.

Data sharing statement Participant information sheets and consent forms are available at https://www.anzctr.org.au/Trial/Registration/TrialReview.aspx?id= 373168\&isReview=true.

Open access This is an open access article distributed in accordance with the Creative Commons Attribution Non Commercial (CC BY-NC 4.0) license, which permits others to distribute, remix, adapt, build upon this work non-commercially, and license their derivative works on different terms, provided the original work is properly cited, appropriate credit is given, any changes made indicated, and the use is non-commercial. See: http://creativecommons.org/licenses/by-nc/4.0/.

\section{REFERENCES}

1. American Psychiatric Association. Diagnostic and Statistical Manual of Mental Disorders, Fifth Edition (DSM-5). Arlington, VA: American Psychiatric Publisher, 2013.

2. Lawlor PG, Gagnon B, Mancini IL, et al. Occurrence, causes, and outcome of delirium in patients with advanced cancer: a prospective study. Arch Intern Med 2000;160:786-94.

3. Hosie A, Davidson PM, Agar M, et al. Delirium prevalence, incidence, and implications for screening in specialist palliative care inpatient settings: A systematic review. Palliat Med 2013;27:486-98.

4. O'Malley G, Leonard M, Meagher D, et al. The delirium experience: a review. J Psychosom Res 2008;65:223-8.

5. Finucane AM, Lugton J, Kennedy C, et al. The experiences of caregivers of patients with delirium, and their role in its management in palliative care settings: an integrative literature review. Psychooncology 2017;26:291-300.

6. National Institute for Health and Clinical Excellence (NICE) National Clinical Guideline centre. Delirium: diagnosis, prevention and management. 2010 http://www.nice.org.uk/nicemedia/live/13060/ 49908/49908.pdf (Accessed 13 Jul 2011).

7. Australian Commission on Safety and Quality in Health Care Evidence for the safety and care of patients with a cognitive impairment in acute care settings: a rapid review. Sydney: ACSQHC, 2013.

8. Leslie DL, Marcantonio ER, Zhang Y, et al. One-year health care costs associated with delirium in the elderly population. Arch Intern Med 2008:168:27-32.

9. Siddiqi N, Harrison JK, Clegg A, et al. Interventions for preventing delirium in hospitalised non-ICU patients. Cochrane Database Syst Rev 2016;3:CD005563.

10. Neufeld KJ, Yue J, Robinson TN, et al. Antipsychotic Medication for Prevention and Treatment of Delirium in Hospitalized Adults: A Systematic Review and Meta-Analysis. J Am Geriatr Soc 2016;64:705-14.

11. Agar MR, Lawlor PG, Quinn S, et al. Efficacy of Oral Risperidone, Haloperidol, or Placebo for Symptoms of Delirium Among Patients in Palliative Care: A Randomized Clinical Trial. JAMA Intern Med 2017;177:34-42.

12. Lawlor PG, Davis DHJ, Ansari M, et al. An analytical framework for delirium research in palliative care settings: integrated epidemiologic, clinician-researcher, and knowledge user perspectives. J Pain Symptom Manage 2014;48:159-75.

13. Hosie A, Agar M, Lobb E, et al. Improving delirium recognition and assessment for people receiving inpatient palliative care: a mixed methods meta-synthesis. Int J Nurs Stud 2017;75:123-9.

14. Hshieh TT, Yue J, Oh E, et al. Effectiveness of multicomponent nonpharmacological delirium interventions: a meta-analysis. JAMA Intern Med 2015;175:512-20.

15. Hosie A, Amgarth-Duff I, Edwards L, et al. Non-pharmacological delirium interventions for adult inpatients with advanced, progressive illness: a systematic review. Nashville, TN: American Delirium Society 7th Annual Meeting, 2017.
16. Gagnon P, Allard P, Gagnon B, et al. Delirium prevention in terminal cancer: assessment of a multicomponent intervention. Psychooncology 2012;21:187-94.

17. Lonergan E, Luxenberg J, Areosa Sastre A, et al. Benzodiazepines for delirium. Cochrane Database of Systematic Reviews 2009;298.

18. Caraceni A. Drug-associated delirium in cancer patients. EJC Supp/ 2013;11:233-40.

19. Hosie A, Lobb E, Agar M, et al. Nurse perceptions of the Nursing Delirium Screening Scale in two palliative care inpatient units: a focus group study. J Clin Nurs 2015;24:3276-85.

20. Wright DK, Brajtman S, Cragg B, et al. Delirium as letting go: An ethnographic analysis of hospice care and family moral experience. Palliat Med 2015;29:959-66.

21. Higginson IJ, Booth S. The randomized fast-track trial in palliative care: role, utility and ethics in the evaluation of interventions in palliative care? Palliat Med 2011;25:741-7.

22. University of Wollongong. Palliative Care Outcomes Collaborative. 2018 http://www.pcoc.org.au/.

23. Gaudreau JD, Gagnon P, Harel F, et al. Fast, systematic, and continuous delirium assessment in hospitalized patients: the nursing delirium screening scale. J Pain Symptom Manage 2005;29:368-75.

24. Trzepacz PT, Mittal D, Torres R, et al. Validation of the Delirium Rating Scale-revised-98: comparison with the delirium rating scale and the cognitive test for delirium. [erratum appears in $\mathrm{J}$ Neuropsychiatry Clin Neurosci 2001 Summer;13(3):433]. Journal of Neuropsychiatry \& Clinical Neurosciences 2001;13:229-42.

25. Virdun C, Luckett T, Davidson PM, et al. Dying in the hospital setting A systematic review of quantitative studies identifying the elements of end-of-life care that patients and their families rank as being most important. Palliat Med 2015;29:774-96.

26. Australian Commission on Quality and Safety of Healthcare. Delirium Clinical Care Standard. 2017 https://www.safetyandquality.gov.au/ our-work/clinical-care-standards/delirium-clinical-care-standard/ (Accessed 20 Aug 2018).

27. Michie S, van Stralen MM, West R. The behaviour change wheel: a new method for characterising and designing behaviour change interventions. Implement Sci 2011;6:42

28. Teodorczuk A, Mukaetova-Ladinska E, Corbett S, et al. Learning about the patient: an innovative interprofessional dementia and delirium education programme. Clin Teach 2014;11:497-502.

29. Walsh A. An exploration of Biggs' constructive alignment in the context of work-based learning. Assessment \& Evaluation in Higher Education 2007;32:79-87.

30. Abernethy AP, Shelby-James T, Fazekas BS, et al. The Australiamodified Karnofsky Performance Status (AKPS) scale: a revised scale for contemporary palliative care clinical practice [ISRCTN81117481]. BMC Palliat Care 2005;4:7.

31. Fries BE, Schneider DP, Foley WJ, et al. Refining a casemix measure for nursing homes. Resource Utilisation Groups (RUG-III) Medical Care 1994;32:668-85.

32. Masso M, Allingham SF, Banfield M, et al. Palliative Care Phase: inter-rater reliability and acceptability in a national study. Palliat Med 2015;29:22-30.

33. Gaudreau JD, Gagnon P, Harel F, et al. Impact on delirium detection of using a sensitive instrument integrated into clinical practice. Gen Hosp Psychiatry 2005;27:194-9.

34. Grassi L, Caraceni A, Beltrami E, et al. Assessing delirium in cancer patients: the Italian versions of the Delirium Rating Scale and the Memorial Delirium Assessment Scale. J Pain Symptom Manage 2001;21:59-68.

35. Leonard M, Raju B, Conroy M, et al. Reversibility of delirium in terminally ill patients and predictors of mortality. Palliat Med 2008;22:848-54.

36. Leonard M, Spiller J, Keen J, et al. Symptoms of depression and delirium assessed serially in palliative-care inpatients. Psychosomatics 2009;50:506-14.

37. Meagher DJ, Morandi A, Inouye SK, et al. Concordance between DSM-IV and DSM-5 criteria for delirium diagnosis in a pooled database of 768 prospectively evaluated patients using the delirium rating scale-revised-98. BMC Med 2014;12:164

38. Sepulveda E, Franco JG, Trzepacz PT, et al. Performance of the Delirium Rating Scale-Revised-98 Against Different Delirium Diagnostic Criteria in a Population With a High Prevalence of Dementia. Psychosomatics 2015;56:530-41.

39. Liamputtong P, Ezzy D. In-depth Interviews. Qualitative Research Methods. 2nd edn. South Melbourne: Oxford University Press, 2005

40. Cocks K, Torgerson DJ. Sample size calculations for pilot randomized trials: a confidence interval approach. J Clin Epidemiol 2013;66:197-201.

41. Harris PA, Taylor R, Thielke R, et al. Research electronic data capture (REDCap)--a metadata-driven methodology and workflow process 
for providing translational research informatics support. J Biomed Inform 2009;42:377-81.

42. Chan AW, Tetzlaff JM, Altman DG, et al. SPIRIT 2013 statement: defining standard protocol items for clinical trials. Ann Intern Med 2013;158:200-7.

43. Boutron I, Moher D, Altman DG, et al. Extending the CONSORT statement to randomized trials of nonpharmacologic treatment: explanation and elaboration. Ann Intern Med 2008;148:295-309.

44. Campbell MK, Piaggio G, Elbourne DR, et al. Consort 2010 statement: extension to cluster randomised trials. $B M J$ 2010;2012:e5661.

45. Tong A, Sainsbury P, Craig J. Consolidated criteria for reporting qualitative research (COREQ): a 32-item checklist for interviews and focus groups. Int J Qual Health Care 2007;19:349-57. 